University of Wollongong

Research Online

Faculty of Engineering and Information

Faculty of Engineering and Information

Sciences - Papers: Part A

Sciences

2014

Improvements on an authentication scheme for vehicular sensor networks

Joseph K. Liu

University of Bristol

Tsz Hon Yuen

University of Hong Kong, thy738@uow.edu.au

Man Ho Au

University of Wollongong, aau@uow.edu.au

Willy Susilo

University of Wollongong, wsusilo@uow.edu.au

Follow this and additional works at: https://ro.uow.edu.au/eispapers

Part of the Engineering Commons, and the Science and Technology Studies Commons

Research Online is the open access institutional repository for the University of Wollongong. For further information contact the UOW Library: research-pubs@uow.edu.au 


\title{
Improvements on an authentication scheme for vehicular sensor networks
}

\begin{abstract}
In a recent paper, Shim (2012) presented a very interesting authentication scheme for vehicular sensor networks. Shim claimed that the scheme is secure against the highest adopted level of attack, namely the chosen-message attack (CID-CMA). Nevertheless, we find that the proof in Shim's paper does not actually prove that the scheme is secure in this level. Instead, it can only ensure that the scheme is secure in a strictly weaker level of attack, the adaptive chosen-identity and no-message attack (CID-NMA). In this paper, first we show that there exist some security risks in vehicular networks if a scheme, which is only secure against CID-NMA but not CID-CMA, is deployed. Hence, having the proof that the scheme is only CID-NMA is insufficient for the aforementioned application. That is, Shim did not prove that the proposed scheme can resist these kinds of attack. Here, we use a different approach to prove the scheme for security against CID-CMA. We note that this proof is essential to ensure that the scheme can indeed be used for the aforementioned scenario. In addition, we also show that the batch verification of the scheme, proposed in the same paper, may have non-negligible error. Two invalid signatures may give a positive result. We further improve the batch verification part so that the error rate can be reduced to negligible level.
\end{abstract}

\section{Keywords}

improvements, authentication, vehicular, sensor, scheme, networks

Disciplines

Engineering | Science and Technology Studies

\section{Publication Details}

Liu, J. K., Yuen, T. Hon., Au, M. \& Susilo, W. (2014). Improvements on an authentication scheme for vehicular sensor networks. Expert Systems with Applications, 41 (5), 2559-2564. 


\title{
Improvements on an Authentication Scheme for Vehicular Sensor Networks
}

\author{
Joseph K. Liu \\ Institute for Infocomm Research, Singapore \\ Tsz Hon Yuen \\ University of Hong Kong, Hong Kong \\ Man $\mathrm{Ho} \mathrm{Au}$ \\ Centre for Computer and Information Security Research \\ School of Computer Science and Software Engineering \\ University of Wollongong, Australia \\ Willy Susilo \\ Centre for Computer and Information Security Research \\ School of Computer Science and Software Engineering \\ University of Wollongong, Australia
}

\begin{abstract}
In a recent paper, Shim [21] presented a very interesting authentication scheme for vehicular sensor networks. Shim claimed that the scheme is secure against the highest adopted level of attack, namely the chosen-message attack (CIDCMA). Nevertheless, we find that the proof in Shim's paper does not actually prove that the scheme is secure in this level. Instead, it can only ensure that the scheme is secure in a strictly weaker level of attack, the adaptive chosenidentity and no-message attack (CID-NMA). In this paper, first we show that there exist some security risks in vehicular networks if a scheme, which is only secure against CID-NMA but not CID-CMA, is deployed. Hence, having the proof that the scheme is only CID-NMA is insufficient for the aforementioned application. That is, Shim did not prove that the proposed scheme can resist these kinds of attack. Here, we use a different approach to prove the scheme for security against CID-CMA. We note that this proof is essential to ensure that the scheme can indeed be used for the aforementioned scenario. In addition, we also show that the batch verification of the scheme, proposed in the same paper, may have non-negligible error. Two invalid signatures may give a positive result. We further improve the batch verification part so that the error rate can
\end{abstract}

\footnotetext{
Email address: ksliu@i2r.a-star.edu.sg, thyuen@cs.hku.hk, \{aau,wsusilo\}@uow.edu.au (Willy Susilo)
} 
be reduced to negligible level.

Keywords: Authentication, Vehicular Sensor Networks

\section{Introduction}

With the advancement of mobile technology, wireless networks have become widely available. Car manufactures and telecommunication industries have started to equip vehicles with wireless devices for interconnection. Cars can communicate with other cars or the roadside infrastructure to improve driving safety or exchange traffic information. These types of vehicular communication networks are usually referred as vehicular ad hoc networks (VANETs). Within the infrastructure of VANETs, privacy and security are the two major challenges. No driver wants to broadcast his/her real identity and current location while in contrast, authentication is required at the same time. Otherwise, one may send some wrong messages or pretend others to send messages. There are many schemes in the literature (such as $[24,15,14,23]$ ) that deal with these two seeming contradictory requirements.

Recently, Shim [21] proposed a conditional privacy-preserving authentication scheme for vehicular sensor networks. It is based on an Identity-based Signature (IBS) scheme proposed in the same paper. Shim adopted the security definition and model of the IBS schemes in [20]. The security model is the normal existential unforgeable against adaptive chosen-identity and chosen-message attack (CID-CMA), which is considered to be the strongest security notion of IBS scheme. Under this notion, the adversary is allowed to query an extraction oracle and a signing oracle. When it submits an identity to the extraction oracle, it returns a private key corresponding to this identity. When it submits a message and an identity to the signing oracle, it returns a valid signature corresponding to this message and identity. After adaptively querying these oracles, the adversary outputs a challenged identity, a challenged message and a valid signature corresponding to this identity and message. The restriction is that: the adversary is not allowed to query the challenged identity to the extraction oracle and the challenged identity-message pair to the signing oracle. However, it is allowed to query the signing oracle for the challenged identity with other messages.

On the other side, in an adaptive chosen-identity and no-message attack (CID-NMA), it is similar to CID-CMA except that no signing oracle is provided. In other words, the adversary is not allowed to see any signature corresponding to the challenged identity. If it happens to see a signature (of any message) from this identity, it may produce some forged signatures or pretend to be this identity to sign some messages. It is a strictly weaker security notion for IBS scheme. If a scheme can only achieve CID-NMA security, it should not be used in general except in the case that the identity can only produce one signature in the whole life time (e.g. one-time signature $[3,19])$.

This Work. The contribution of this paper can be categorized as follow: 
1. We find that there is a flaw in the proof of the IBS in [21]. Although it is claimed to be CID-CMA, we show that the proof of the corresponding theorem cannot attend the claimed security. Instead, only a strictly weaker security, the CID-NMA can be achieved.

2. We describe a list of security risks in VANETs for deploying an authentication scheme which is only CID-NMA secure. In other words, if the scheme in [21] is used, it cannot prove that it can resist such kinds of attacks.

3. We attempt to provide the correct proof for Shim's IBS using a completely different approach. In our proof, we show that the IBS scheme in [21] is CID-CMA secure.

4. In addition, Shim also deployed a batch verification on the signature scheme. We demonstrate that the false acceptance rate is non-negligible: we can easily construct two invalid signatures such that when they are batched together for verification, they become valid signatures. That is, they can pass through the batch verification equation. We further modify the batch verification part to reduce this false acceptance to a negligible level, which is our final contribution in this paper.

\section{Related Works}

We discuss some of the related works here and explain why our improved version has some advancement over existing works.

In the area of security and privacy of Vehicular Ad Hoc Networks (VANETs), a number of research works have been done on anonymous authentication to ensure security and privacy. A majority of these schemes make use of pseudonyms (e.g. $[4,24,13])$ or anonymous credentials (e.g. [9, 12]). A recent approach is to use signature-based technique (e.g. $[16,7]$ ) to achieve anonymous authentication. All these schemes are suitable for authorization. However, these pseudonym-based authentication schemes are prone to generate a huge revocation list, as pointed out in [21]. Another approcah is to deploy group signature (e.g. $[17,18,22])$ to achieve anonymous authentication. But the verification cost in group-signature-based schemes is too expensive for devices in VANETs which may require very fast verification time. Similar to a group signature, a ring signature $[6,25,1]$ can also be used to provide privacy preserving capability. By removing the need for a group manager and allowing a signer to create an adhoc group membership, a ring signature scheme can be used for in applications with the competing requirements of message authenticity and signer privacy.

However, facing the same obstacle as group signature, the verification of ring signature is not efficient enough. On the other side, identity-based schemes (e.g. $[26,21])$ allow fast or batch verification that is particularly suitable for vehicular communications. Nevertheless, the scheme in [26] required the long-term system master key preloaded into all tamper-proof devices and the security rely on it. In practice, these tamper-proof devices may be subjected to side-channel attacks. The compromised of one device results in the leakage of the master 
secret key which is a serious security flaw in the whole system. The scheme in [21] does not contain this risk, though there is a flaw in their security proof and their batch verification is not always sound, as we mentioned in the last section.

We summarize the comparison among these cryptographic primitives in Table 2 .

\begin{tabular}{|c|c|c|c|c|c|}
\hline Primitive & $\begin{array}{c}\text { Efficient } \\
\text { Revocation }\end{array}$ & $\begin{array}{c}\text { Efficient } \\
\text { Verification }\end{array}$ & $\begin{array}{c}\text { No tamper } \\
\text { resist. device }\end{array}$ & $\begin{array}{c}\text { Batch } \\
\text { verification }\end{array}$ & $\begin{array}{c}\text { Correct } \\
\text { Proof }\end{array}$ \\
\hline Pseudonym & $\times$ & $\times$ & $\checkmark$ & $\times$ & $\checkmark$ \\
Group signature & $\times$ & $\times$ & $\checkmark$ & $\times$ & $\checkmark$ \\
Ring signature & $\checkmark$ & $\times$ & $\checkmark$ & $\times$ & $\checkmark$ \\
IBS [26] & $\checkmark$ & $\checkmark$ & $\times$ & $\checkmark$ & $\checkmark$ \\
IBS [21] & $\checkmark$ & $\checkmark$ & $\checkmark$ & $x^{\mathrm{a}}$ & $\times$ \\
Ours & $\checkmark$ & $\checkmark$ & $\checkmark$ & $\checkmark$ & $\checkmark$ \\
\hline
\end{tabular}

${ }^{\mathrm{a}}$ We will later show that the batch verification of [21] is not always correct.

Table 2. Comparison

\section{Security Risks in VANETs for deploying an insecure scheme}

In this section, we present some concrete security risks in VANETS if an insecure scheme (or not secure enough in an acceptable level) is deployed as the underlying security primitive.

\subsection{Deploying a scheme which is only CID-NMA Secure - Message Forgery}

We first demonstrate that by incorporating an Identity-based Signature (IBS) scheme, which is only CID-NMA secure, in VANETs, then some practical security risks in the whole system may be presented.

In the following, we consider the same scenario as in [21]. There are some communications between the road side unit (RSU) and vehicles. When the RSU sends an authenticated message to vehicles, it uses the underlying IBS scheme to sign a message. Upon receiving the message, the vehicle verifies the signature corresponding to the RSU. If the scheme is only CID-NMA secure but not CID-CMA secure, the vehicle cannot ensure that the received signature is really signed by the RSU. This is because an adversary can pretend to be the RSU to generate a valid message (once it has seen a valid signature by the RSU), and hence, the authenticity is lost.

Additionally, when a vehicle sends an authenticated message to the RSU, it signs the message with his/her pseudo-identity. When an adversary obtains this signature from this pseudo-identity, it can produce another valid signature for this pseudo-identity within the valid time period although it does not have its private key. This may result in a message forgery. The adversary can then deliberately send false and harmful messages using this pseudo-identity as it is no longer accountable to the adversary but the pseudo-identity of the victim. This results in the security breakdown of the whole system. 


\subsection{False Acceptance on Batch Verification}

In a batch verification, the whole batch would be dropped or rejected, even if there is just one false signature in the batch. It should be a deterministic process with no exception. Otherwise, a harmful message may get through the batch verification process (that is, the authentication gateway) to jeopardize the safety of the traffic system. An adversary may take advantage of this loophole by injecting some harmful messages in each authentication cycle. This attack may bring fatal traffic consequences for a VANET-based traffic system.

Roadmap. In the next section, we will show that the proof for the IBS in [21] only shows that the scheme is secure against CID-NMA but not CID-CMA. That is, Shim [21] did not prove that the proposed authentication scheme can resist against the attack mentioned above. Due to the lack of this proof, then the scheme in [21] may not be able to be adopted in the VANET scenario mentioned in the original paper. Fortunately, in Section 5, we are able to provide the correct proof so that the scheme achieves CID-CMA.

Subsequently, we show that the batch verification in [21] contains nonnegligible false acceptance rate in Section 6 . We then propose a fix to the batch verification process which can prevent the attack mentioned above.

\section{Brief Review of the IBS scheme proposed in [21]}

In this section, we briefly review the IBS scheme proposed in [21] and point out the problem in the security proof. We first review some mathematical tools used.

We denote the security parameter by $k$. Suppose $\mathbb{G}$ and $\mathbb{G}_{T}$ are groups of prime order $q$. We write the group operation in $\mathbb{G}$ additively ${ }^{1}$. The function $e: \mathbb{G} \times \mathbb{G} \rightarrow \mathbb{G}_{T}$ is a bilinear pairing such that $\forall P, Q \in \mathbb{G}$ and $a, b \in \mathbb{Z}_{q}, e(a P, b Q)=$ $e(P, Q)^{a b} ; e(P, P)$ generates $\mathbb{G}_{T}$ if $g$ is a generator of $\mathbb{G}$. We require that the group operations in $\mathbb{G}$ and $\mathbb{G}_{T}$, and the bilinear pairing $e$ are computable in polynomial time with respect to $k$. Let $\mathcal{G}\left(1^{k}\right)$ be a probabilistic polynomial time (PPT) algorithm which outputs $\left(\mathbb{G}, \mathbb{G}_{T}, q, P, e\right)$, where $P$ is the generator of $\mathbb{G}$ and $e: \mathbb{G} \times \mathbb{G} \rightarrow \mathbb{G}_{T}$ is a bilinear pairing.

The following Computational Diffie-Hellman (CDH) assumption is used in [21].

Definition 1 (CDH assumption). Given g, $x P, y P \in \mathbb{G}$ where $\mathbb{G}$ is a group of prime order $q$ and $x, y$ are randomly chosen from $\mathbb{Z}_{q}$, no adversary can output $x y P$ in polynomial time $t$ with non-negligible probability $\epsilon$. We denote it as the $(t, \epsilon)-C D H$ assumption.

\footnotetext{
${ }^{1}[21]$ used the multiplicative notation in their definition but used the additive notation in the rest of the paper.
} 


\subsection{The Scheme}

Shim [21] firstly proposed a standard signature scheme $\mathcal{K S S}$ which is summarized as follow:

- Setup. On input the security parameter $k$, run $\left(\mathbb{G}, \mathbb{G}_{T}, q, P, e\right) \leftarrow \mathcal{G}\left(1^{k}\right)$ where $e: \mathbb{G} \times \mathbb{G} \rightarrow \mathbb{G}_{T}$ is the bilinear pairing defined above such that $\mathbb{G}$ and $\mathbb{G}_{T}$ are groups of order $q$ and $P$ is a generator of $\mathbb{G}$. Choose a cryptographic hash function $H_{1}:\{0,1\}^{*} \rightarrow \mathbb{Z}_{q}$. The public parameters are param $=\left(\mathbb{G}, \mathbb{G}_{T}, q, P, e, H_{1}\right)$.

- KeyGen. On input param, pick a random $x \in_{R} \mathbb{Z}_{q}^{*}$ and $Q \in_{R} \mathbb{G}$. Compute $Y=x P$ and set $P K=(Y, Q)$ as the public key and $S K=x$ as the secret key.

- Sign. On input param, $S K$ and a message $m \in\{0,1\}^{*}$, choose a random number $k \in \mathbb{Z}_{q}^{*}$. Compute $T=k P, h=H_{1}(m, T)$ and $S=(x+h k) Q$. Output the signature $\sigma=(T, S)$.

- Verify. On input param, $P K, m$ and $\sigma=(T, S)$, compute $h=H_{1}(m, T)$ and check if $e(S, P)=e(Y+h T, Q)$. If it holds, accept the signature.

Shim [21] adopted the definition and formal security model of existential unforgeable against adaptive chosen message attack (EUF-CMA) of standard signatures in [11]. The author has also shown that the signature scheme $\mathcal{K S S}$ is EUF-CMA secure if the CDH assumption holds.

Shim [21] proposed an identity-based signature scheme $\mathcal{K I B S}$ which is based on $\mathcal{K S S}$. Note that the extract algorithm of $\mathcal{K} \mathcal{I B S}$ is the same as the signing algorithm of $\mathcal{K S S}$ with the identity $I D$ as message.

The scheme is described as follow:

- Setup. On input the security parameter $k$, run $\left(\mathbb{G}, \mathbb{G}_{T}, q, P, e\right) \leftarrow \mathcal{G}\left(1^{k}\right)$ where $e: \mathbb{G} \times \mathbb{G} \rightarrow \mathbb{G}_{T}$ is the bilinear pairing defined above such that $\mathbb{G}$ and $\mathbb{G}_{T}$ are groups of order $q$ and $P$ is a generator of $\mathbb{G}$. Pick two more generators $Q, Q^{\prime} \in \mathbb{G}$ and a random $s \in_{R} \mathbb{Z}_{q}^{*}$. Compute $P_{p u b}=s P$. Choose two cryptographic hash functions $H_{1}:\{0,1\}^{*} \rightarrow \mathbb{Z}_{q}$ and $H_{2}:\{0,1\}^{*} \rightarrow \mathbb{Z}_{q}$. The public parameters are param $=\left(\mathbb{G}, \mathbb{G}_{T}, q, P, Q, Q^{\prime}, P_{\text {pub }}, e, H_{1}, H_{2}\right)$. The master secret key is $s$.

- Extract. On input param, $s$ and an identity $I D \in\{0,1\}^{*}$, choose a random number $k \in \mathbb{Z}_{q}^{*}$. Compute $T_{I D}=k P, h=H_{1}\left(I D, T_{I D}\right)$ and $S_{I D}=(s+h k) Q$. Output the private key $s k_{I D}=\left(T_{I D}, S_{I D}\right)$.

- IB-Sign. On input param, $s k_{I D}$, a message $M \in\{0,1\}^{*}$, choose a random number $r \in \mathbb{Z}_{q}^{*}$. Compute $U=r P, h^{\prime}=H_{2}\left(I D, M, T_{I D}, U\right)$ and $V=$ $h^{\prime} S_{I D}+r Q^{\prime}$. Output the signature $\tau=\left(T_{I D}, U, V\right)$.

- IB-Verify. On input param, $I D, M$ and $\tau=\left(T_{I D}, U, V\right)$, compute $h=$ $H_{1}\left(I D, T_{I D}\right), h^{\prime}=H_{2}\left(I D, M, T_{I D}, U\right)$ and check if

$$
e(V, P)=e\left(h^{\prime} \cdot\left[P_{p u b}+h T_{I D}\right], Q\right) \cdot e\left(U, Q^{\prime}\right) .
$$


If it holds, accept the signature.

\subsection{The Proof}

Shim [21] proved that if $\mathcal{K S S}$ is EUF-CMA secure, then $\mathcal{K I B S}$ is secure under the adaptive chosen identity and chosen message attack (CID-CMA). CID-CMA security means that the adversary is allowed to ask for the private keys of arbitrary identities and signatures of arbitrary messages. The adversary wins if it can output a signature $\tau^{*}$ for a message $M^{*}$ and an identity $I D^{*}$, such that the private key of $I D^{*}$ was not asked and no signature on $M^{*}$ for $I D^{*}$ was asked.

We find that the security proof of Theorem 3.2 in [21] only showed that $\mathcal{K I B S}$ is secure under the adaptive chosen identity and no message attack (CIDNMA). It is different from the CID-CMA that in the case of CID-NMA, no signature on any message for $I D^{*}$ was allowed to ask by the adversary (the adversary can still ask for the signature for other identities).

In the proof in [21], Shim simulated the IB-Sign query by requesting an Extract query to $\mathcal{K S S}$ to obtain a private key, and then used it to run the IBSign algorithm. Therefore, if the adversary asked an IB-Sign query on $I D^{*}$ and some message $M$, it forces the simulator to request an Extract query on $I D^{*}$. At the end of the security game, even if the adversary outputs a forgery and the simulator extracts the private key of $I D^{*}$, the simulator still cannot use it as a forgery of the $\mathcal{K S S}$. It is because the simulator has asked this private key before (during the IB-Sign query). Therefore, the simulator in the proof in [21] cannot handle such IB-Sign query on $I D^{*}$ and hence it only achieves CID-NMA security.

Another way to see the invalidity of the proof is the omission of $H_{2}$ in the security proof of $\mathcal{K} \mathcal{I B S}$. In the proof of $\mathcal{K S S}, H_{1}$ is used for rewinding in the Forking Lemma and hence we require that $H_{1}$ to be a cryptographic hash function. On the other hand, no specific property of $H_{2}$ is needed for the security proof of $\mathcal{K} \mathcal{I B S}[21]$. It implies that $H_{2}$ can be any arbitrary function, without affecting the security. It is obviously incorrect: a collision on $\mathrm{H}_{2}$, such as $H_{2}\left(I D, M_{1}, T_{I D}, U\right)=H_{2}\left(I D, M_{2}, T_{I D}, U\right)$ for some $M_{1} \neq M_{2}$, will result in a forgery under the adaptive chosen message attack. Therefore, we should at least require that $H_{2}$ is collision resistant in the security proof.

\section{Proof for CID-CMA secure}

In this section, we provide a formal proof that $\mathcal{K} \mathcal{I B S}$ is CID-CMA secure assuming $\mathcal{K S S}$ is EUF-CMA secure using a different approach from the original paper [21]. Looking ahead, our proof and Shim's proof both follows the reduction approach. The difference is that we show how to handle the signature query on the challenge identity.

A typical reduction proof goes as follows. We assume on the contrary that $\mathcal{K I B S}$ is not CID-CMA secure. That is, assume there exists an attacker $\mathcal{A}$ that can forge a signature in $\mathcal{K} \mathcal{I B S}$ under the adaptive chosen identity and chosen 
message attack. The proof's goal is to show that under this assumption, $\mathcal{K S} \mathcal{S}$ is not EUF-CMA secure. We said we have reduced the security of $\mathcal{K} \mathcal{I} \mathcal{S}$ to the security of $\mathcal{K S S}$, for if the former is insecure, so is the latter. Specifically, we need to show the existence of another algorithm $\mathcal{S}$ that, with the help of $\mathcal{A}$, breaks to EUF-CMA security of $\mathcal{K S \mathcal { S }}$. Reduction proof in cryptography is often constructive, that is, we show how to construct the algorithm $\mathcal{S}$ based on the algorithm $\mathcal{A}$.

proof: Let $\mathcal{A}$ be a forger of $\mathcal{K} \mathcal{I B S}$ under the definition of CID-CMA security. We show how to construct a forger $\mathcal{S}$ against $\mathcal{K S} \mathcal{S}$ under the definition of EUFCMA security.

Under the definition of EUF-CMA security, $\mathcal{S}$ is given the public parameter and public key of an instance of the $\mathcal{K S S}$ scheme. That is, it receives as input $\left(\mathbb{G}, \mathbb{G}_{T}, q, P, e, H_{1}, Y, Q\right)$. $\mathcal{S}$ 's goal is to output a message $m^{*}$ and a forged signature $\sigma^{*}=\left(T^{*}, S^{*}\right)$ of $m^{*}$ that passes the verification algorithm. That is,

$$
h=H_{1}\left(m^{*}, T^{*}\right) \wedge e\left(S^{*}, P\right)=e(Y+h T, Q) .
$$

In addition, as an EUF-CMA attacker, $\mathcal{S}$ can freely obtain signatures of any messages of its choice. To model this, we say $\mathcal{S}$ is given an oracle, usually called a signing oracle, $\mathcal{O}_{\mathcal{K S S}}$ that on input $m$, output a pair $(S, T)$ such that

$$
h=H_{1}(m, T) \wedge e(S, P)=e(Y+h T, Q) .
$$

For notational convenience, we shall denote the operation of the signing oracle as $(S, T) \leftarrow \mathcal{O}_{\mathcal{K S S}}(m)$. Of course, the natural restriction is that $m^{*}$ cannot be an input to $\mathcal{O}_{\mathcal{K S S}}$.

Below we show how $\mathcal{S}$ produces the forgery with the help of $\mathcal{A}$.

- Setup. $\mathcal{S}$ creates the public parameter and public key of an instance of $\mathcal{K I B S}$ as follows. It randomly selects a value $\alpha \in_{R} \mathbb{Z}_{q}$, computes $Q^{\prime}=Q^{\alpha}$. It also chooses a hash function $H_{2}$. The public parameter is set as:

$$
\text { param }=\left(\mathbb{G}, \mathbb{G}_{T}, q, P, e, P_{\text {pub }}:=Y, Q, Q^{\prime}, H_{1}, H_{2}\right) .
$$

param is given to $\mathcal{A}$ as the public parameter of an instance of $\mathcal{K} \mathcal{I S S}$.

- Queries. As an CID-CMA attacker, $\mathcal{A}$ is entitled to freely obtain signing key corresponds to any identity $I D$ under the restriction that $I D$ is not the identity of the forged signature. Furthermore, $\mathcal{A}$ is allowed to obtain the signature of any message on any identity. Again, the forgery should not be on the message-identity tuple that has been queried. Below we show how $\mathcal{S}$ provides the signing keys and signatures requested by $\mathcal{A}$. One point to note is that in the random oracle model, $\mathcal{A}$ cannot compute the hash values of $H_{2}$ by itself. It has to be obtained from $\mathcal{S}$.

- $H_{2}$ Query. $\mathcal{A}$ requests the hash value of the tuple $\left(v_{1}, v_{2}, v_{3}, v_{4}\right) . \mathcal{S}$ maintains a list $\mathcal{L}$ for the hash values issued so far. If $\left(v_{1}, v_{2}, v_{3}, v_{4}\right)$ does not exist in the list $\mathcal{L}, \mathcal{S}$ returns a random value $h^{\prime} \epsilon_{R} \mathbb{Z}_{q}$. 
$h^{\prime}$ is put on $\mathcal{L}$ with the tuple $\left(v_{1}, v_{2}, v_{3}, v_{4}\right)$. On the other hand, if $\left(v_{1}, v_{2}, v_{3}, v_{4}\right)$ already exists in the list $\mathcal{L}$, the corresponding $h^{\prime}$ is returned. This maintain the consistency of the hash function and at the same time ensure the hash function $H_{2}$ acts as a random function.

- Extraction Query. $\mathcal{A}$ requests the signing key for identity $I D$. $\mathcal{S}$ issues $(S, T) \leftarrow \mathcal{O}_{\mathcal{K S S}}(I D)$. $\mathcal{S}$ returns $\left(S_{I D}:=S, T_{I D}=T\right)$ to $\mathcal{A}$ as the signing key of identity $I D$.

- Signature Query. $\mathcal{A}$ requests the signature for identity ID on message $m$. Note that the following is different to the original proof from [21]. We extend the proof to handle the case when $\mathcal{S}$ does not know the signing key of an identity $I D$. This is necessary in CID-CMA security because $\mathcal{A}$ can request signatures for the challenge identity for different messages. If there exists an extraction query with input $I D$, $\mathcal{S}$ simply creates the signature using the corresponding signing key $\left(S_{I D}, T_{I D}\right)$. On the other hand, if $I D$ has never been an input of the extraction query, $\mathcal{S}$ does the following.

1. If this is the first time this $I D$ is involved, $\mathcal{S}$ randomly generates $T_{I D} \in_{R} \mathbb{G}$, Otherwise, re-use the existing value of $T_{I D}$.

2. Compute $h=H_{1}\left(I D, T_{I D}\right)$.

3. Generate at random $h^{\prime}, k \in_{R} \mathbb{Z}_{q}$.

4. Compute $V=k Q$.

5. Compute $U=\alpha^{-1}\left(k P-h^{\prime}\left(P_{p u b}+h T_{I D}\right)\right)$.

6. Add $\left(I D, m, T_{I D}, U\right), h^{\prime}$ to the list $\mathcal{L}$. Note that this implicitly sets $H_{2}\left(I D, m, T_{I D}, U\right)$ to have the value $h^{\prime}$. The probability that the tuple $\left(I D, m, T_{I D}, U\right)$ already exists in $\mathcal{L}$ is negligible since the value $U$ depends on the random value $k$ and is freshly generated for each signature query.

$\mathcal{S}$ returns $\left(T_{I D}, U, V\right)$ as the signature on message $m$ under identity ID.

- Forgery. Finally, $\mathcal{A}$ outputs a forgery $\left(m^{*}, I D^{*}, T_{I D^{*}}^{*}, U^{*}, V^{*}\right)$ (with some non-negligible probability) such that

$$
\begin{aligned}
h & =H_{1}\left(I D^{*}, T_{I D^{*}}^{*}\right) \\
h^{\prime} & =H_{2}\left(I D^{*}, m^{*}, T_{I D^{*}}^{*}, U^{*}\right) \\
e\left(V^{*}, P\right) & =e\left(h^{\prime} \cdot\left[P_{p u b}+h T_{I D^{*}}^{*}\right], Q\right) \cdot e\left(U^{*}, Q^{\prime}\right) .
\end{aligned}
$$

- General Forking Lemma [2]. Let $\epsilon$ be the probability that $\mathcal{A}$ outputs a valid forgery. Due to the general forking lemma, there exists a forking algorithm that can obtain another tuple $\left(m^{*}, I D^{*}, T_{I D^{*}}^{*}, U^{*}, \bar{V}^{*}\right)$ from $\mathcal{A}$ (in another run of $\mathcal{A}$ using the same random coins for $\mathcal{A}$ ) such that

$$
\begin{aligned}
h & =H_{1}\left(I D^{*}, T_{I D^{*}}^{*}\right) \\
\overline{h^{\prime}} & =H_{2}\left(I D^{*}, m^{*}, T_{I D^{*}}^{*}, U^{*}\right) \\
e\left(\bar{V}^{*}, P\right) & =e\left(\bar{h}^{\prime} \cdot\left[P_{p u b}+h T_{I D^{*}}^{*}\right], Q\right) \cdot e\left(U^{*}, Q^{\prime}\right)
\end{aligned}
$$


with probability greater than

$$
\epsilon(\epsilon /|\mathcal{L}|-1 / q)
$$

Note that $|\mathcal{L}|$ is the size of the list $\mathcal{L}$ which is the total number of hash query made by $\mathcal{A}$ and that $\overline{h^{\prime}} \neq h^{\prime}$. (Essentially, the forking algorithm runs $\mathcal{A}$ twice, with the same random tape for $\mathcal{A}$ and that the hash queries are answered differently from the point where $\mathcal{A}$ issues the hash query for $\left(I D^{*}, m^{*}, T_{I D^{*}}^{*}, U^{*}\right)$.)

- Output of $\mathcal{S}$. $\mathcal{S}$ computes $S^{*}=\left(h^{\prime}-\bar{h}^{\prime}\right)^{-1}\left(V^{*}-\bar{V}^{*}\right)$, sets $T^{*}=T_{I D^{*}}^{*}$ and $m^{*}=I D^{*}$ and outputs $\left(S^{*}, T^{*}\right)$ as the forgery on message $m^{*}$ in the scheme $\mathcal{K S S}$.

\section{Batch Verification}

In this section, we first review the batch verification part of the authentication scheme in [21]. Then we point out some cases such that when two invalid signatures are batched verified, the algorithm outputs a positive result.

\subsection{The original batch verification in [21]}

The authentication scheme basically is the same as the underlying IBS KIBS scheme, except that the identity $I D$ is replaced by the pseudo-identity PID. Hence we only review the batch verification part.

On input param and $n$ distinct message-signature tuples $\left\langle P I D_{1}, M_{1}, t t_{1}, \tau_{1}\right\rangle$, $\ldots,\left\langle P I D_{n}, M_{n}, t t_{n}, \tau_{n}\right\rangle$, where $\tau_{i}=\left(T_{P I D_{i}}, U_{i}, V_{i}\right)$ and $t t_{i}$ is the current timestamp, the RSU performs the following procedures:

1. Compute $h_{i}=H_{1}\left(P I D_{i}, T_{P I D_{i}}\right)$ and $h_{i}^{\prime}=H_{2}\left(P I D_{i}, M_{i}, t t_{i}, T_{P I D_{i}}, U_{i}\right)$ for $i=1, \ldots, n$.

2. Verify whether

$$
e\left(\sum_{i=1}^{n} V_{i}, P\right)=e\left(\left[\sum_{i=1}^{n} h_{i}^{\prime}\right] \cdot P_{p u b}+\sum_{i=1}^{n} h_{i}^{\prime} h_{i} T_{P I D_{i}}, Q\right) \cdot e\left(\sum_{i=1}^{n} U_{i}, Q^{\prime}\right)
$$

holds or not. If it holds, accept the signatures. ${ }^{2}$

\subsection{False Acceptance}

Next, we show that when two invalid signatures are batched together for verification, equation (2) gives a positive result.

Assume $\tau_{1}=\left(T_{P I D_{1}}, U_{1}, V_{1}\right)$ and $\tau_{2}=\left(T_{P I D_{2}}, U_{2}, V_{2}\right)$ are two valid signatures on the message-identity tuples $\left\langle P I D_{1}, M_{1}, t t_{1}\right\rangle$ and $\left\langle P I D_{2}, M_{2}, t t_{2}\right\rangle$ respectively.

\footnotetext{
${ }^{2}$ We note that there is a typo in the original paper in [21]. The first term on the right hand side of the equation $\left[\sum_{i=1}^{n} h_{i}^{\prime}\right]$ was wrongly written as $\left[\sum_{i=1}^{n} h_{i}\right]$. We correct the typo here.
} 
That is, they pass through equation (1) for individual verification. Definitely they can also pass through equation (2) for batch verification.

Now we change the signatures to $\tau_{1}^{\prime}=\left(T_{P I D_{1}}, U_{1}, V_{1}^{\prime}\right)$ and $\tau_{2}^{\prime}=\left(T_{P I D_{2}}, U_{2}, V_{2}^{\prime}\right)$ where $V_{1}^{\prime}=V_{1}+a \cdot P$ and $V_{2}^{\prime}=V_{2}-a \cdot P$ for some $a \in \mathbb{Z}_{q}^{*}$. Obviously, $\left\langle P I D_{1}, M_{1}, t t_{1}, \tau_{1}^{\prime}\right\rangle$ and $\left\langle P I D_{2}, M_{2}, t t_{2}, \tau_{2}^{\prime}\right\rangle$ cannot pass through equation (1). In other words, these two signatures are invalid. However, when they are batched together for verification, they can pass through equation (2). This is due to the term $a \cdot P$ in $V_{1}^{\prime}$ has been canceled by the term $-a \cdot P$ in $V_{2}^{\prime}$.

\subsection{An Improved batch verification}

This kind of false acceptance can be avoided by adding the well-known small exponents test $([8,10,5])$. The patch is as follow:

1. For batch verifying $n$ signatures, choose $\delta_{i} \epsilon_{R}\{0,1\}^{\ell}$ for $i=1, \ldots, n$, where $\ell$ is a security parameter such that the probability of accepting a bad pair is $2^{-\ell}$. (The false acceptance rate was proven as Theorem 3.1 in [10]. Readers may refer to that paper for the details.) The size of $\ell$ is a tradeoff between efficiency and security. Usually $\ell=80$ should be enough for normal scenario in VANETs.

2. Compute $h_{i}=H_{1}\left(P I D_{i}, T_{P I D_{i}}\right)$ and $h_{i}^{\prime}=H_{2}\left(P I D_{i}, M_{i}, t t_{i}, T_{P I D_{i}}, U_{i}\right)$ for $i=1, \ldots, n$.

3. Verify whether

$$
e\left(\sum_{i=1}^{n} \delta_{i} \cdot V_{i}, P\right)=e\left(\sum_{i=1}^{n}\left(h_{i}^{\prime} \cdot \delta_{i}\right) \cdot\left(P_{p u b}+h_{i} T_{P I D_{i}}\right), Q\right) \cdot e\left(\sum_{i=1}^{n} \delta_{i} \cdot U_{i}, Q^{\prime}\right)
$$

holds or not. If it holds, accept the signatures.

We note that an attack such as what we illustrated earlier will no longer be applicable after applying our patch as described above.

\section{Conclusion}

In this paper, we showed that the underlying IBS scheme in [21] cannot achieve an acceptable security level. The risks are twofold: (i) the proof of the IBS scheme in [21] can only ensure that the scheme is secure against CID-NMA, which is a strictly weaker security level than the normal CID-CMA; (ii) the batch verification part of the authentication scheme in [21] has a non-negligible false acceptance rate. We also showed that by deploying a scheme with the above risks, there exists some practical implications on security in VANETs. Finally, we provide improvements to fix the two loopholes so that the IBS should be secure enoguh to be used in VANETs.

There are two future research directions: The first one is to develop an IBS with batch verification without pairing. Pairing is a costly mathematical algorithm that requires relatively expensive computation power. Although batch verification can greatly reduce the number of pairing operations, we still require 
3 pairing in our improvement. We leave it as a future research to construct an IBS with batch verification without pairing so that lightweight device such as smart card can easily execute. By then, it will give more flexibility for engineers or researchers to design the infrastructure of VANET. The second one is to shorten the length of the signature. Currently the signature consists of three group elements. If the signature can be further shorten, the communication cost will be reduced accordingly.

\section{Acknowledgement}

W. Susilo is supported by the ARC Future Fellowship (FT0991397) and ARC Discovery Project (DPDP130101383).

\section{References}

[1] M. H. Au, J. K. Liu, W. Susilo, and T. H. Yuen. Secure id-based linkable and revocable-iff-linked ring signature with constant-size construction. Theor. Comput. Sci., 469:1-14, 2013.

[2] M. Bellare and G. Neven. Multi-signatures in the plain public-key model and a general forking lemma. In CCS 2006, pages 390-399. ACM, 2006.

[3] K. Bicakci, G. Tsudik, and B. Tung. How to construct optimal one-time signatures. Computer Networks, 43(3):339-349, 2003.

[4] G. Calandriello, P. Papadimitratos, J.-P. Hubaux, and A. Lioy. Efficient and robust pseudonymous authentication in VANET. In Vehicular Ad Hoc Networks, pages 19-28. ACM, 2007.

[5] J. Camenisch, S. Hohenberger, and M. Ø. Pedersen. Batch verification of short signatures. J. Cryptology, 25(4):723-747, 2012.

[6] B. K. Chaurasia and S. Verma. Conditional privacy through ring signature in vehicular ad-hoc networks. Transactions on Computational Science, 13:147-156, 2011.

[7] L. Chen, S.-L. Ng, and G. Wang. Threshold Anonymous Announcement in VANETs. IEEE Journal on Selected Areas in Communications, 29(3):605$615,2011$.

[8] J. H. Cheon and D. H. Lee. Use of sparse and/or complex exponents in batch verification of exponentiations. IEEE Trans. Computers, 55(12):1536-1542, 2006.

[9] T. W. Chim, S.-M. Yiu, L. C. K. Hui, and V. O. K. Li. OPQ: OT-Based Private Querying in VANETs. IEEE Transactions on Intelligent Transportation Systems, 12(4):1413-1422, 2011. 
[10] A. L. Ferrara, M. Green, S. Hohenberger, and M. Ø. Pedersen. Practical short signature batch verification. In CT-RSA, volume 5473 of Lecture Notes in Computer Science, pages 309-324. Springer, 2009.

[11] S. Goldwasser, S. Micali, and R. L. Rivest. A digital signature scheme secure against adaptive chosen-message attacks. SIAM J. Comput., 17(2):281-308, 1988.

[12] A. Gonzalez-Tablas, A. Alcaide, J. de Fuentes, and J. Montero. Privacypreserving and accountable on-the-road prosecution of invalid vehicular mandatory authorizations. In Ad Hoc Networks, pages ??-?? Elsevier, 2013.

[13] D. Huang, S. Misra, M. Verma, and G. Xue. PACP: An Efficient Pseudonymous Authentication-Based Conditional Privacy Protocol for VANETs. IEEE Transactions on Intelligent Transportation Systems, 12(3):736-746, 2011.

[14] D. Huang, S. Misra, M. Verma, and G. Xue. PACP: An Efficient Pseudonymous Authentication-Based Conditional Privacy Protocol for VANETs . IEEE Transactions on Intelligent Transportation Systems, 12(3):736-746, 2011.

[15] J.-L. Huang, L.-Y. Yeh, and H.-Y. Chien. ABAKA: An Anonymous Batch Authenticated and Key Agreement Scheme for Value-Added Services in Vehicular Ad Hoc Networks . IEEE Transactions on Vehicular Technology, 60(1):248-262, 2011.

[16] G. Kounga, T. Walter, and S. Lachmund. Proving reliability of anonymous information in VANETs. IEEE Trans. Vehicular Technology, 58(6):29772989, 2009.

[17] X. Lin, X. Sun, P.-H. Ho, and X. Shen. GSIS: Secure vehicular communications with privacy preserving. IEEE Trans. Vehicular Technology, 56(6):3442-3456, 2007.

[18] X. Lin, X. Sun, P. H. Ho, and X. Shen. Security in vehicular ad hoc networks. IEEE Comm. Mag., 46(4):88-95, 2008.

[19] P. Mohassel. One-time signatures and chameleon hash functions. In Selected Areas in Cryptography, volume 6544 of Lecture Notes in Computer Science, pages 302-319. Springer, 2010.

[20] K.-A. Shim. An ID-based aggregate signature scheme with constant pairing computations. Journal of Systems and Software, 83(10):1873-1880, 2010.

[21] K.-A. Shim. $\mathcal{C P} \mathcal{A S}$ : An Efficient Conditional Privacy-Preserving Authentication Scheme for Vehicular Sensor Networks. IEEE Transactions on Vehicular Technology, 61(4):1874-1883, 2012. 
[22] J. Sun, C. Zhang, Y. Zhang, and Y. Fang. An identity-based security system for user privacy in vehicular ad hoc networks. IEEE Trans. Parallel Distrib. Syst., 21(9):1227-1239, 2010.

[23] Y. Sun, Z. Feng, Q. Hu, and J. Su. An efficient distributed key management scheme for group-signature based anonymous authentication in VANET. Security and Communication Networks, 5(1):79-86, 2012.

[24] Y. Sun, R. Lu, X. Lin, X. Shen, and J. Su. An efficient pseudonymous authentication scheme with strong privacy preservation for vehicular communications. IEEE Transactions on Vehicular Technology, 59(7):3589-3603, 2010 .

[25] T. H. Yuen, J. K. Liu, M. H. Au, W. Susilo, and J. Zhou. Efficient linkable and/or threshold ring signature without random oracles. Comput. J., 56(4):407-421, 2013.

[26] C. Zhang, R. Lu, X. Lin, P.-H. Ho, and X. Shen. An efficient identity-based batch verification scheme for vehicular sensor networks. In INFOCOM, pages 246-250. IEEE, 2008. 